\title{
Modelo de vínculo relacional de egresados con una universidad regional del estado en Chile
}

\author{
Segundo R. Cabana, Felicindo H. Cortés, Isidora V. Farías, y Rodrigo P. Véliz \\ Universidad de La Serena, Facultad de Ingeniería, Dpto. de Ing. Industrial, Casilla 554, La Serena-Chile \\ (correo-e: rcabana@userena.cl; fcortes@userena.cl; ifarias@rebuss.cl; rveliz@exa-digital.com)
}

Recibido Ene. 27, 2021; Aceptado Mar. 24, 2021; Versión final May. 21, 2021, Publicado Oct. 2021

\begin{abstract}
Resumen
El objetivo de esta investigación es identificar y analizar las variables que influyen en el vínculo relacional entre una universidad regional chilena y sus egresados. Para esto, se realizó un estudio basado en la técnica de ecuaciones estructurales a través del método de mínimos cuadrados parciales (PLS, en inglés) desarrollando el caso particular de la Universidad de La Serena. Esto permitió construir un modelo causal de seis hipótesis con los siguientes constructos: 1) educación continua, 2) satisfacción del egresado, 3) calidad de enseñanza, 4) confianza de la red de apoyo, 5) fidelidad del egresado y 6 ) identificación del egresado. Se concluye con un modelo causal con un buen ajuste $(0,531)$ y todas sus variables significativas, permitiendo establecer una fuerte influencia del constructo satisfacción de los egresados en el vínculo relacional que poseen hacia su alma máter.
\end{abstract}

Palabras clave: satisfacción; fidelidad; vínculo relacional; ecuaciones estructurales; egresados

\section{Model of relational bond of graduates with a regional public Chilean university}

\begin{abstract}
The objective of this research study is to identify and analyze the variables that influence the relational bond between a Chilean regional university and its graduates. For this, a study based on the technique of structural equations is performed through the partial least squares (PLS) method, examining the specific case of the University of La Serena. This allows developing a causal model of six hypotheses with the following constructs: 1) continuing education, 2) graduate satisfaction, 3) teaching quality, 4) support network trust, 5) graduate loyalty, and 6) graduate identification. In conclusion, a causal model with a good fit (0.531) and all its significant variables is developed, allowing to establish a strong influence of graduates' construct satisfaction for the relational bond they have towards their alma mater.
\end{abstract}




\section{INTRODUCCIÓN}

Como todo tipo de organizaciones, el desarrollo de las instituciones de educación superior depende de una estrategia que asegure su calidad y mantenga su responsabilidad social, siendo capaces de incluir la excelencia en el desarrollo de competencias, manteniendo altos estándares que generen valor y satisfacción en los interesados (Shams y Belyaeva, 2019). Es por ello que las estrategias de las instituciones de educación superior se deben centrar en mejorar la satisfacción del estudiante y su fidelidad, estimulando al estudiante a que forme un vínculo con su alma mater (Cabana et al., 2016). Este vínculo permite que los estudiantes tomen un rol activo haciéndolos partícipes de los cambios en los procesos de enseñanza de la organización, y si ese vínculo perdura en el tiempo más allá del término de las relaciones contractuales, el egresado se transforma en un socio clave que permite mejorar la calidad de la educación, el prestigio y la reputación de la universidad, incluso puede facilitar la adquisición de tecnología minimizando los problemas financieros y mejorando la competitividad de la organización (Iskhakova et al., 2016).

Este paradigma de gestión educacional, donde la calidad de la educación es tanto una meta a corto plazo como un objetivo estratégico (Cabana et al., 2016), es prioritario y trascendental para Chile, dado el momento histórico que vive el país y la intensa discusión en torno a la educación que se ha instalado en la sociedad chilena dado el proceso constituyente que se ha iniciado. A esto se suma la pandemia provocada por el COVID - 19, que ha calado hondo en las 59 universidades estatales chilenas colocando en jaque su sistema de financiamiento. En este contexto, la Universidad de La Serena (ULS), única institución de educación superior del estado de Chile en la Región de Coquimbo y una de las universidades más importantes del país, perteneciente al consejo de rectores (CRUSH), está envuelta en un problema significativo dada su estructura de ingresos, la cual se distribuye en un $83,2 \%$ de recursos propios y a un aporte fiscal directo de un $3,37 \%$, transformando a la ULS en una organización que funciona como una institución estatal, con toda su burocracia y limitaciones, pero con un sistema de ingresos casi de una empresa privada, realidad que se repite en la gran mayoría de universidades estatales chilenas.

Si bien, existe una amplia cantidad de estudios que proponen modelos para medir la satisfacción o fidelidad de los estudiantes y/o egresados de establecimientos de educación superior, con el fin de resolver problemas similares a los padecidos por la ULS, muchas veces estos se enfocan en variables específicas y/o los modelos deben de ser validados. Tal es el caso del modelo de Satisfacción-Lealtad de Doña y Luque (2020) donde han podido correlacionar ambas variables acotando su investigación a estudiantes del ámbito económicoempresarial, o como el modelo basado en ecuaciones estructurales Intention to Alumni Loyalty (IAL) presentado por Iskhakova et al. (2016), que según sus autores, otorga un enfoque integrador que permite superar algunas de las limitaciones de los modelos parciales cuando se trata de explicar la intención de la fidelidad de los egresados, pero carece de la aplicabilidad en otros países dado que, según las conclusiones de su investigación aplicada en estudiantes de universidades de Alemania y Rusia, existen diferencias entre países que influyen significativamente en la intención de la fidelidad de los egresados, incluso, los resultados podrían diferir entre universidades privadas y públicas. En esta misma línea, los autores Schlesinger et al. (2015), presentan un modelo que determina los elementos claves que construyen la relación egresado universidad, tomando como variable principal la identificación del egresado con su alma mater, pero con las mismas limitaciones que las investigaciones anteriores. Así una serie de investigaciones no solo tienen esta limitación, sino que también no abordan el "vínculo" como un constructo a estudiar, lo cuál implica un cambio de enfoque e innovación en esta investigación.

Dado el escenario anterior y la situación en la que se encuentra la ULS, es imperativo determinar un modelo validado en un entorno con características socio-culturales a fines de la Universidad de La serena, que permita posicionar y mantener, financieramente estable, a la Universidad de La Serena, manteniendo y/o superando las expectativas de la sociedad y sus alumnos, que una vez egresados, mantengan altos niveles de satisfacción, incentivando su participación e involucramiento a través del tiempo, originando así, una educación continua, sostenible y sustentable de la universidad. Además de propiciar un modelo replicable a otras instituciones de educación superior de similares características. Finalmente, el objetivo de esta investigación es encontrar un modelo causal que permita comprender y analizar las diferentes variables que influyen en el vínculo relacional con los egresados, por lo que se proponen seis hipótesis. En vista del escenario anterior se desarrolla el sustento teórico de las hipótesis que se presentan en la Tabla 1 y que conforman el modelo conceptual propuesto representado en la Figura 1.

Tabla 1: Hipótesis de investigación

\begin{tabular}{|c|l|}
\hline Hipótesis & Descripción \\
\hline H1 & La educación continua influye directa y positivamente en la satisfacción del egresado \\
\hline H2 & La calidad de enseñanza influye directa y positivamente en la satisfacción del egresado \\
\hline H3 & La satisfacción del egresado influye directa y positivamente en la fidelidad del egresado \\
\hline H4 & La confianza en la red de apoyo influye directa y positivamente en la fidelidad del egresado \\
\hline H5 & La identificación del egresado influye directa y positivamente en la fidelidad del egresado \\
\hline H5 & La fidelidad del egresado influye directa y positivamente en el vínculo relacional del egresado. \\
\hline
\end{tabular}




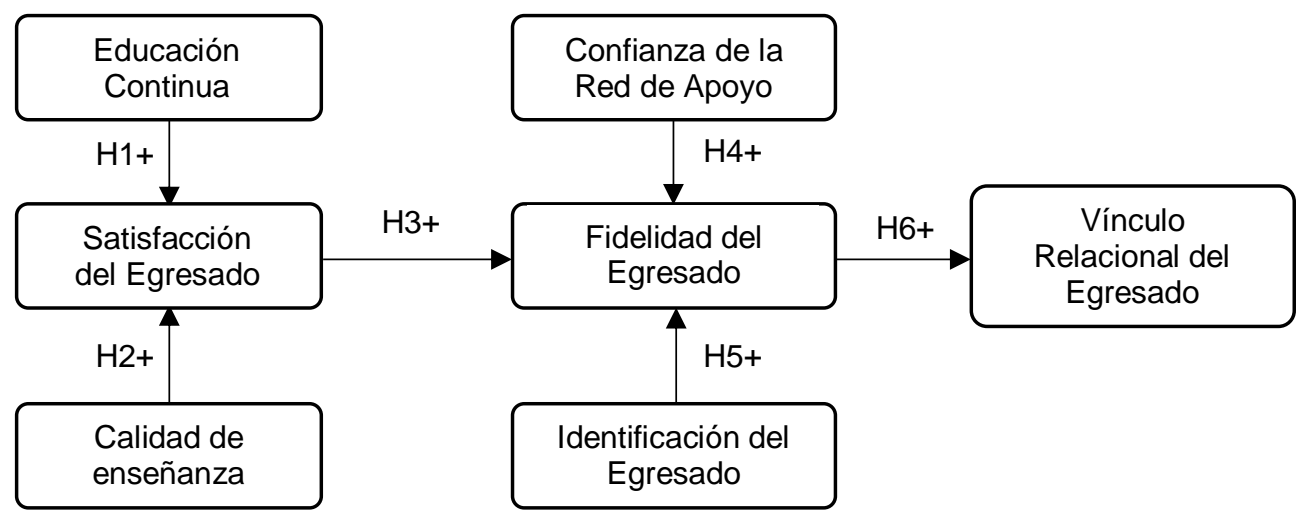

Fig. 1: Modelo conceptual

\section{OTROS ANTECEDENTES}

En el actual escenario educativo de alta competitividad, emprendedor e innovador, las universidades se ven obligadas a mantener un ritmo cada vez más acelerado, sufriendo en el camino los embates de estos cambios inevitables. En este escenario se propone un modelo que permita maximizar los beneficios de la relación entre los egresados y su alma mater, la cual al ser bidireccional (tanto la institución como el estudiante se benefician) genera un vínculo relacional que se puede ver afectado por los siguientes constructos:

\section{Educación continua del egresado, calidad de enseñanza y satisfacción}

La importancia de la educación continua en la contribución al perfeccionamiento profesional de los egresados, es determinante, por ello las universidades invierten recursos en el seguimiento de sus egresados analizando su inserción en el mercado laboral, con el fin de mejorar sus procesos de enseñanza y formación, manteniendo una oferta actualizada de las demandas que, tanto los alumnos y la sociedad, mantienen respecto de la vida universitaria (Gutiérrez et al., 2011). Es por ello que la educación continua debe ser capaz de ofrecer información y entregar oportunidades de capacitación o perfeccionamiento en la especialidad, generando la necesidad en las universidades de asegurar las capacidades y servicios profesionales, más allá de la obtención del título por parte del egresado, permitiendo así satisfacer, no tan solo la demanda social y de mercado en cuanto a la formación general y especializada. Sino que también satisfacer al egresado, posibilitando continuar con el vínculo hacia un postgrado y/o mantener un contacto a largo plazo con la universidad (Gibson, 2010), lo que lleva a proponer la siguiente hipótesis: H1: La educación continua influye directa y positivamente en la satisfacción del egresado.

Por otro lado, la calidad de servicio, per se, es muy difícil de definir y medir, existiendo un amplio debate en la comunidad científica, pero llegando a un acuerdo general en que la calidad de servicio percibida es una forma de actitud relacionada, pero que no es equivalente, con la satisfacción del "cliente-estudiante" (Douglas et al., 2008; Cabana et al., 2016) Donde el valor de la educación superior percibido por el estudiante deriva sobre todo de la relación precio / calidad (valor funcional), valor epistémico, en términos de calidad de la enseñanza y el valor funcional asociado con ganancias futuras y objetivos profesionales (Alves, 2011). En esta línea, se puede apreciar que la satisfacción es un indicador clave de la calidad en el servicio educativo prestado por una institución pública de educación superior. En consecuencia, la calidad percibida y la satisfacción se hallan estrechamente ligadas entre sí, por lo que, a mayor calidad, existiría una mayor satisfacción de los egresados, es por ello que las estrategias centradas en mejorar la satisfacción del estudiante y su fidelidad se deben convertir en decisiones estratégicas en los centros de educación superior (CES), donde la calidad de la educación es simultáneamente una meta de corto plazo como también un objetivo estratégico (Cabana et al., 2016). Por tanto: H2: La calidad de enseñanza influye directa y positivamente en la satisfacción del egresado.

Entendiendo la satisfacción del egresado como una respuesta cognitiva y afectiva generada después de vivir la experiencia como estudiante, esta refleja la eficiencia de los servicios académicos y administrativos de la institución (Schlesinger et al., 2014). Al ser la experiencia positiva, se genera un cierto nivel de continuidad en la relación del alumno con la institución, un vínculo psicológico o emocional representado por la promesa explícita o implícita de continuar eligiendo los servicios de la institución e incluso recomendándola, mejorando la imagen y reputación de la universidad en su entorno (Schlesinger et al., 2012). Es por ello que los estudiantes satisfechos tienen más probabilidades de mantener una fidelidad hacia la institución que los ha formado, continuando los enlaces durante el postgrado y estando más predispuestos a mantener contacto con la universidad luego de su graduación (Doña y Luque, 2020), demostrando que la satisfacción tiene un impacto significativo en la fidelidad (Zaheer y Rehman, 2010). En sentido, es importante identificar el valor 
percibido en satisfacción de los estudiantes y egresados ya que, al ser un valor epistémico y emocional, se condiciona las posibilidades de desarrollo de los egresados en su etapa de estudiantes si no se tienen en cuenta sus propias expectativas, intereses y gustos, condicionando la satisfacción final. Esta satisfacción, no solo tiene una importancia trascendental al transformar a egresados en "embajadores" que posicionen a la misma universidad, sino que también son una pieza angular para la mejora continua, motivando a los egresados a aportar su visión crítica con el fin de alterar estructuras y orientaciones curriculares y mejorar los servicios complementarios que ofrece la universidad, proporcionando educación y competencias profesionales sintonizadas con los requerimientos del mercado laboral (Casanoves et al., 2019; Doña y Luque 2020). Esto implica que: H3: La satisfacción del egresado influye directa y positivamente en la fidelidad del egresado.

\section{Confianza de la red de apoyo, identificación del egresado y fidelidad del egresado}

La confianza es una variable clave para mejorar el alcance de una relación afectando el compromiso con la universidad (Schlesinger et al., 2016; Frasquet et al., 2012), el cual está determinado en gran medida por el grado de integración que tuvo el estudiante en el sistema universitario a través de la participación activa en sociedades universitarias, a través de su grupo de amistades y conocidos participando en actividades extracurriculares de la universidad e interactuar con otros estudiantes de distintas instituciones (Iskhakova et al., 2016). Esta imagen construida desde el entorno del egresado influye en las percepciones que tiene de la institución (Schlesinger et al., 2016), incluso, la exposición a influencias grupales, como miembros de la familia, padres o amigos puede conducir a una mayor propensión a hacer contribuciones y apoyos caritativos (Iskhakova et al., 2016), formando un fuerte vínculo y compromiso y por consecuencia fidelidad con su institución de educación, lo que finalmente permite formular la siguiente hipótesis: H4: La confianza de la red de apoyo influye directa y positivamente en la fidelidad del egresado.

La identificación de un ex alumno con su universidad, se conceptualiza como el grado en que los miembros de la organización se perciben a sí mismos y a la universidad como un conjunto que comparte los mismos atributos y valores, en un intento de satisfacer una o más necesidades personales e institucionales (Dutton et al., 1994). Esta percepción de la organización hace que los individuos se sientan vinculados con la organización, lo cual sumado al prestigio o reputación de la misma, influye directamente en los niveles de identificación del estudiante y egresado, siendo capaces de defenderla y sentirse parte de ella, aumentando la intención de elegirla de nuevo para estudios de postgrado, seguir en contacto con ella, recomendarla o hablar bien de ella en su entorno (Scott y Lane, 2000). En este sentido Schlesinger et al. (2012) plantea que la identificación de los egresados conlleva un compromiso psicológico y una preocupación por la organización que los motiva a sentirse comprometidos con sus objetivos, colaborar activamente e interactuar y cooperar con sus miembros, estableciendo una relación más estrecha y a largo plazo con la misma (Bhattacharya y Sen, 2003). Este apego emocional o identificación juegan un papel fundamental en la generación de fidelización del egresado con su universidad, proporcionando una relación sólida que permite un vínculo a largo plazo (Iskhakova et al., 2016), lo que implica: H5: La identificación del egresado influye directa y positivamente en la fidelidad del egresado.

\section{Fidelidad y vínculo relacional del egresado con su universidad}

Complementando lo expuesto anteriormente sobre la fidelidad del egresado, esta posee tanto un componente actitudinal como de comportamiento, y es en este último donde se aprecian las conductas de vinculación con la institución de educación (Ali et al., 2016). De hecho, Helgesen y Nesset (2007) argumentan que la fidelidad de un egresado puede ser más importante que el de un estudiante actual, aplicándose a cualquier universidad, curso en particular o cualquier otro componente de la universidad, porque la fidelidad de un egresado no es un efecto a corto plazo, pues crea un vínculo que lo podría llevar a integrarse y colaborar con su desarrollo. Después de obtener sus títulos, los egresados pueden convertirse en fuertes defensores de su universidad, así como en los niveles de identificación, de recomendar la institución a otros o proseguir con su aprendizaje continuamente (Schlesinger et al., 2015), manteniendo un vínculo a largo plazo que permita seguir construyendo relaciones, es por ello y dado los actuales desafíos de la educación superior, es necesario que las organizaciones de educación integren de forma planificada al estudiante en la co-creación de innovaciones en procesos de apoyo y en los servicios educacionales (Cabana et al., 2018). H6: La fidelidad del egresado influye directa y positivamente en el vínculo relacional del egresado.

\section{METODOLOGÍA}

Con el fin de analizar las relaciones entre los distintos constructos, y así corroborar las hipótesis planteadas en el modelo causal, se presenta un estudio empírico, donde se aplica una encuesta a una muestra de 384 egresados de la Universidad de La Serena, Chile, con un universo desconocido y un 95\% de confiabilidad. La investigación de carácter exploratorio se realizó con el software estadístico Smart PLS y el software IBM SPSS Statistics 23. En la Tabla 2 se entregan detalles de la ficha técnica de investigación. 
Tabla 2: Ficha técnica de investigación

\begin{tabular}{|c|c|c|c|c|}
\hline \multicolumn{2}{|c|}{ Datos generales del muestreo } & \multicolumn{3}{|c|}{ Datos descriptivos de la muestra } \\
\hline \multirow{2}{*}{ Tipo de Muestreo } & \multirow{2}{*}{ Muestreo Aleatorio Simple } & \multirow{4}{*}{ Rango de Edad } & $20-25$ años & $39,8 \%$ \\
\hline & & & $26-30$ años & $41,4 \%$ \\
\hline \multirow[b]{2}{*}{ Nivel de confianza } & \multirow{2}{*}{$\begin{array}{l}95 \% ; z=1,96 ; p=q=0,5(5 \% \\
\text { error y } 95 \% \text { de confiabilidad })\end{array}$} & & $31-35$ años & $12,3 \%$ \\
\hline & & & $\begin{array}{l}36 \text { años o } \\
\text { mayores }\end{array}$ & $6,5 \%$ \\
\hline Universo de egresados & Desconocido & \multirow{2}{*}{ Género } & Masculino & $42,1 \%$ \\
\hline Tamaño muestral & 384 & & Femenino & $57,9 \%$ \\
\hline Unidad de análisis & Región de Coquimbo, Chile & \multirow{3}{*}{$\begin{array}{l}\text { Tipo de Institución de } \\
\text { Enseñanza Media del que } \\
\text { egresó }\end{array}$} & Municipal & $22,3 \%$ \\
\hline $\begin{array}{l}\text { Método de recogida de } \\
\text { la información }\end{array}$ & $\begin{array}{l}\text { Cuestionario presencial y } \\
\text { online de } 26 \text { preguntas }\end{array}$ & & Subvencionado & $72,5 \%$ \\
\hline $\begin{array}{l}\text { Tipo de pregunta } \\
\text { encuesta }\end{array}$ & $\begin{array}{l}\text { Politómicas tipo Likert (1 a 5) } \\
\text { de apreciación numérica }\end{array}$ & & Particular & $5,2 \%$ \\
\hline $\begin{array}{l}\text { Fecha de realización } \\
\text { de trabajo de campo }\end{array}$ & $\begin{array}{l}\text { Entre noviembre } 2018 \text { y abril } \\
2019\end{array}$ & $\begin{array}{l}\text { Rango año de defensa de } \\
\text { título }\end{array}$ & $2010-2$ & \\
\hline
\end{tabular}

\section{Análisis de fiabilidad individual de los indicadores}

En primera instancia, para determinar la fiabilidad de los indicadores, fue necesario calcular las cargas factoriales de cada una de las variables de los constructos aceptando las cargas factoriales con valores superiores a 0,6 (Moriano et al., 2009), bajo este criterio se eliminaron los indicadores DC2, SE3, CR3 y VR1, el resto de los indicadores se consideran aceptables y significativos al 95\%. Además, se realizó la prueba de Kaiser-Meyer-Olkin (KMO), considerándose adecuado cuando es mayor que 0,6 y la prueba de esfericidad de Bartlett que prueba la hipótesis nula de que las variables están incorrelacionadas, se acepta como válido un nivel de significación menor que 5\% (Espinoza et al., 2015), cumpliendo con ambos criterios todos los constructos, como se aprecia en la Tabla 3.

Tabla 3: Análisis de fiabilidad individual de los Indicadores

\begin{tabular}{|c|c|c|c|}
\hline Constructo & Indicador & Ítem & Carga Factorial \\
\hline \multirow{4}{*}{$\begin{array}{l}\text { Educación } \\
\text { Continua } \\
\text { KMO: } 0,698 \\
\text { PEB: } 0,000\end{array}$} & $\mathrm{DC} 1$ & $\begin{array}{l}\text { La Universidad tiene una oferta posteriori a pregrado, pertinente a los } \\
\text { desafíos de la sociedad y mercado }\end{array}$ & 0,861 \\
\hline & DC2 & $\begin{array}{l}\text { Los programas de post grados con los que cuenta La Universidad } \\
\text { son ampliamente conocidos }\end{array}$ & 0,489 \\
\hline & DC3 & $\begin{array}{l}\text { Considero que la Universidad ofrece cursos acordes a lo solicitado } \\
\text { por el mercado actual }\end{array}$ & 0,641 \\
\hline & DC4 & $\begin{array}{l}\text { La Universidad cuenta con buenas instalaciones e infraestructura } \\
\text { para el desarrollo de estudios diferentes a pregrado }\end{array}$ & 0,843 \\
\hline \multirow{2}{*}{$\begin{array}{l}\text { Calidad de } \\
\text { Enseñanza } \\
\text { KMO: } 0,755 \\
\text { PEB: } 0,000\end{array}$} & CE1 & $\begin{array}{l}\text { Los procesos de enseñanza y aprendizaje donde participé me } \\
\text { generaron resultados académicos adecuados }\end{array}$ & 0,796 \\
\hline & CE2 & $\begin{array}{l}\text { Considero que tuve la libertad de decidir y actuar para mejorar los } \\
\text { procesos de enseñanza aprendizaje en la carrera }\end{array}$ & 0,668 \\
\hline \multirow{2}{*}{$\begin{array}{l}\text { Calidad de } \\
\text { Enseñanza } \\
\text { KMO: 0,755 } \\
\text { PEB: } 0,000\end{array}$} & CE3 & $\begin{array}{l}\text { En las clases en general, percibí al profesor como un guía que me } \\
\text { involucraba para mejorar mi aprendizaje con más autonomía }\end{array}$ & 0,819 \\
\hline & CE4 & $\begin{array}{l}\text { Los académicos cumplieron con mis expectativas respecto a las } \\
\text { competencias requeridas para lograr calidad en mi aprendizaje }\end{array}$ & 0,850 \\
\hline \multirow{2}{*}{$\begin{array}{l}\text { Satisfacción } \\
\text { del Egresado } \\
\text { KMO: } 0,729 \\
\text { PEB: } 0,000\end{array}$} & SE1 & Mi decisión de haber seleccionado mi universidad fue la correcta. & 0,893 \\
\hline & SE2 & $\begin{array}{l}\text { Me encuentro satisfecho pues lo que aportó mi universidad } \\
\text { contribuyó a mi desarrollo profesional. }\end{array}$ & 0,884 \\
\hline \multirow{2}{*}{$\begin{array}{l}\text { Satisfacción } \\
\text { del Egresado } \\
\text { KMO: } 0,729 \\
\text { PEB: } 0,000\end{array}$} & SE3 & Lo aprendido contribuyó a mi actual empleabilidad. & $-0,002$ \\
\hline & SE4 & $\begin{array}{l}\text { Me encuentro satisfecho pues lo que aportó mi Universidad } \\
\text { contribuyó a mi desarrollo personal. }\end{array}$ & 0,886 \\
\hline
\end{tabular}


Tabla 3: continuación.

\begin{tabular}{|c|c|c|c|}
\hline Constructo & Indicador & Ítem & Carga Factorial \\
\hline \multirow{4}{*}{$\begin{array}{l}\text { Confianza de } \\
\text { la Red de } \\
\text { Apoyo } \\
\text { KMO: } 0,674 \\
\text { PEB: } 0,000\end{array}$} & CR1 & $\begin{array}{l}\text { Mis actuales redes profesionales de apoyo tienen una imagen } \\
\text { positiva de mi universidad. }\end{array}$ & 0,802 \\
\hline & CR2 & $\begin{array}{l}\text { Mis actuales redes sociales de apoyo tienen una imagen positiva de } \\
\text { mi universidad. }\end{array}$ & 0,646 \\
\hline & CR3 & $\begin{array}{l}\text { En general, la confianza de mis actuales redes de apoyo, se potencia } \\
\text { a través de las reuniones de egresados. }\end{array}$ & 0,544 \\
\hline & CR4 & $\begin{array}{l}\text { En el ámbito laboral donde me desempeño, sus integrantes expresan } \\
\text { opiniones positivas de mi Universidad }\end{array}$ & 0,646 \\
\hline \multirow{3}{*}{$\begin{array}{l}\text { Identificación } \\
\text { del Egresado } \\
\text { KMO: } 0,736 \\
\text { PEB: } 0,000\end{array}$} & IE1 & $\begin{array}{l}\text { Cuando alguien en mi entorno cercano critica a la Universidad, me } \\
\text { duele y lo tomo como si me criticaran }\end{array}$ & 0,912 \\
\hline & IE2 & Cuando la Universidad obtiene un éxito, siento como si fuera mío & 0,897 \\
\hline & IE3 & $\begin{array}{l}\text { Cuando hablo de la Universidad, normalmente uso frases como "Mi } \\
\text { universidad", con actitud de compromiso. }\end{array}$ & 0,932 \\
\hline \multirow{3}{*}{$\begin{array}{l}\text { Fidelidad del } \\
\text { Egresado } \\
\text { KMO: } 0,685 \\
\text { PEB: } 0,000\end{array}$} & FE1 & $\begin{array}{l}\text { Si alguien me pidiera consejos académicos, yo la recomendaría la } \\
\text { Universidad como una primera opción. }\end{array}$ & 0,804 \\
\hline & FE2 & Soy un promotor del potencial transformador de la Universidad. & 0,774 \\
\hline & FE3 & $\begin{array}{l}\text { Estoy dispuesto a colaborar con ideas para la mejora continua de la } \\
\text { universidad. }\end{array}$ & 0,851 \\
\hline \multirow{4}{*}{$\begin{array}{l}\text { Vínculo } \\
\text { Relacional del } \\
\text { Egresado } \\
\text { KMO: } 0,692 \\
\text { PEB: } 0,000\end{array}$} & VR1 & Me siento integrado al desarrollo de la Universidad. & 0,453 \\
\hline & VR2 & $\begin{array}{l}\text { La reputación de mi Universidad me da accesos efectivos a redes y } \\
\text { organizaciones que potencian mi desarrollo. }\end{array}$ & 0,718 \\
\hline & VR3 & $\begin{array}{l}\text { Estoy dispuesto a actuar como un socio colaborador al desarrollo de } \\
\text { la universidad. }\end{array}$ & 0,818 \\
\hline & VR4 & $\begin{array}{l}\text { En mi quehacer laboral y con mis redes profesionales potencio el } \\
\text { capital reputacional de mi universidad }\end{array}$ & 0,851 \\
\hline
\end{tabular}

\section{Fiabilidad y validez del constructo}

A continuación, se analiza la fiabilidad de cada constructo, para ello se calcula el indicador Alpha de Cronbach, el cual se considera aceptable cuando su valor sea mayor a 0,7 (Forcael et al., 2013), comprobándose que todos los constructos de este modelo alcanzan valores adecuados de fiabilidad. Luego, para analizar la consistencia interna de los constructos, se calcula el indicador de Fiabilidad Compuesta, admitiéndose valores superiores a 0,7 (Casanoves et al., 2019), bajo este criterio se corrobora la consistencia interna del modelo.

Finalmente, se evalúa la validez de las escalas utilizadas (validez convergente), para lo cual se analiza la varianza extraída media (AVE). Se recomienda que sea superior a 0,5 (Forcael et al., 2013), cumpliéndose con este requisito en todos los constructos utilizados. En el siguiente Tabla 4 se exponen los resultados de los análisis anteriores.

Tabla 4: Alpha de Cronbach, índice de fiabilidad compuesta y validez convergente

\begin{tabular}{|l|c|c|c|}
\hline & Alfa de Cronbach & Fiabilidad compuesta & Varianza extraída media (AVE) \\
\hline Educación Continua & 0,856 & 0,857 & 0,777 \\
\hline Calidad de Enseñanza & 0,793 & 0,866 & 0,619 \\
\hline Satisfacción del Egresado & 0,721 & 0,841 & 0,641 \\
\hline Confianza de la Red de Apoyo & 0,902 & 0,938 & 0,836 \\
\hline Identificación del Egresado & 0,745 & 0,852 & 0,657 \\
\hline Fidelidad del Egresado & 0,715 & 0,755 & 0,508 \\
\hline Vínculo Relacional del Egresado & 0,738 & 0,850 & 0,654 \\
\hline
\end{tabular}

\section{Validez discriminante}

Luego de analizar la consistencia interna (validez convergente), es necesario calcular la validez discriminante, la cual indica en qué medida un constructo es diferente a los otros que conforman el modelo. Una forma de comprobar este criterio de validación es demostrar que las correlaciones entre los constructos son más bajas que la raíz cuadrada de la varianza extraída media AVE (Forcael et al., 2013). Como se observa en la Tabla 5 , todos los valores cumplen para la validez discriminante. 
Tabla 5: Validez discriminante

\begin{tabular}{|l|c|c|c|c|c|c|c|}
\hline \multicolumn{1}{|c|}{ Constructo } & $D C$ & $C E$ & $S$ & $C R$ & $I$ & $F$ & $V R$ \\
\hline Educación Continua & 0,801 & & & & & & \\
\hline Calidad de Enseñanza & 0,594 & 0,787 & & & & & \\
\hline Satisfacción del Egresado & 0,600 & 0,739 & 0,881 & & & & \\
\hline Confianza de la Red de Apoyo & 0,527 & 0,552 & 0,496 & 0,713 & & & \\
\hline Identificación del Egresado & 0,443 & 0,484 & 0,596 & 0,456 & 0,914 & & \\
\hline Fidelidad del Egresado & 0,620 & 0,623 & 0,756 & 0,544 & 0,595 & 0,811 & \\
\hline Vínculo Relacional del Egresado & 0,544 & 0,605 & 0,731 & 0,526 & 0,728 & 0,744 & 0,809 \\
\hline
\end{tabular}

\section{RESULTADOS}

Con el fin de analizar y confirmar las relaciones existentes entre las variables y la aceptación o rechazo de las hipótesis, se utilizará el método de modelos estructurales a través del Método de Mínimos Cuadrados Parciales (Partial Least Squares, PLS).

\section{Análisis univariante y bivariante}

Los resultados de la encuesta señalan que la muestra se integra en su mayoría por mujeres con un $57,9 \%$ mientras que solo un $42,1 \%$ de hombres respondieron la encuesta, el rango de edad predominante es entre 26 y 30 años $(41,4 \%)$, el $72,5 \%$ de los egresados indicó haber cursado sus estudios de nivel secundario en una institución privada con aportes fiscales (colegios subvencionados), y el 31,4\% de los encuestados defendió su título el año 2017. En cuanto al análisis bivariante, se utilizará la escala de medición contenida en la tabla 6. De la Tabla 6 se entenderá el rango entre 61-70 como nivel "Medio bajo" y el rango entre 71-80 como nivel "Medio alto" para su análisis más detallado.

Tabla 6: Escala de medición

\begin{tabular}{|c|c|c|}
\hline Desde (\%) & Hasta (\%) & Nivel \\
\hline 0 & 20 & Inexistente \\
\hline 21 & 40 & Muy Bajo \\
\hline 41 & 60 & Bajo \\
\hline 61 & 80 & Medio \\
\hline 81 & 90 & Alto \\
\hline 91 & 100 & Muy Alto \\
\hline
\end{tabular}

Los egresados muestran un bajo nivel en cuanto a educación continua $(58,50 \%)$, con una percepción independiente de los factores, aceptando la hipótesis nula dado que $\mathrm{F}$ calculado es menor al $\mathrm{F}$ tabular (Ruiz y Cruz, 2016). Por lo que, género $(0,058<3,87)$, edad $(1,258<2,64)$, institución de educación secundaria $(0,546<3,03)$, año de titulación $(1,008<1,97)$ y carrera $(1,410<1,50)$ no tienen diferencias significativas entre sus medias poblacionales. A nivel de calidad de enseñanza los egresados de la ULS la han catalogado con un nivel medio bajo $(67,10 \%)$, no cumpliendo con las expectativas de los egresados. Esta percepción es totalmente independiente de su edad $(0,106<2,64)$, su institución de educación secundaria $(0,563<3,03)$, su año de titulación $(1,140<1,97)$ y su carrera $(1,188<1,50)$. En una misma línea se encuentra la satisfacción de los egresados con un nivel medio bajo de $69,21 \%$. Esta percepción es totalmente independiente de los factores género $(3,398<3,87)$, edad $(1,720<2,64)$, institución de educación secundaria $(0,680<3,03)$, año de titulación $(1,587<1,97)$ y carrera $(1,179<1,50)$.

La identificación del egresado con la ULS muestra que es baja $(58,50 \%)$, percepción que no depende del género $(0,697<3,87)$, de la edad $(1,801<2,64)$, su institución de educación secundaria $(0,004<3,03)$ o su carrera $(1,069<1,50)$ pero si existen diferencias significativas a nivel de año de titulación $(2,260>1,97)$. La confianza en la red de apoyo que poseen los egresados se encuentra en un nivel medio alto $(71,86 \%)$ donde no existen diferencias significativas en los factores género $(0,379<3,87)$, edad $(0,171<2,64)$, institución de educación secundaria $(1,363<3,03)$, carrera $(0,830<1,50)$ y año de titulación $(0,354<1,97)$. En cuando a la fidelidad del egresado con la ULS esta se encuentra en un nivel medio bajo que alcanza el $67,75 \%$. Esta percepción es independiente del género $(0,697<3,87)$, la edad $(2,158<2,64)$, la institución de educación secundaria $(0,406<3,03)$ y la carrera $(0,974<1,50)$ del egresado, pero existen diferencias significativas a nivel de año de titulación $(2,032>1,97)$. Siguiendo la misma línea de las anteriores variables, el vínculo relacional de los egresados con la ULS es de un nivel medio bajo $(63,17 \%)$ donde solo se observan diferencias significativas en cuanto a año de titulación $(2,561 \mathrm{Fcal}>1,97 \mathrm{Ftab})$. 


\section{Validación del modelo estructural}

Tomando en consideración los análisis anteriores se evalúa las relaciones causales que se proponen en el modelo por medio de dos índices básicos: la varianza explicada (R2) la cual debe ser igual o mayor que 0,1 (Falk y Miller, 1992) y el el Test de Stone-Geisser (Q2) (Johnson et al., 2006), que se usa como criterio para medir la relevancia predictiva de los constructos dependientes. En el caso de que la Q2>0, indica que el modelo tiene relevancia predictiva, en caso contrario, no la tiene (Esposito et al., 2008). Como se aprecia en la Tabla 7, todos los constructos cumplen con ambas reglas.

Tabla 7: Validez del modelo

\begin{tabular}{|l|c|c|}
\hline \multicolumn{1}{|c|}{ Constructo } & $R^{2}$ & $Q^{2}$ \\
\hline Satisfacción del Egresado & 0,587 & 0,428 \\
\hline Fidelidad del Egresado & 0,405 & 0,316 \\
\hline Vínculo Relacional del Egresado & 0,531 & 0,324 \\
\hline
\end{tabular}

Índice de bondad de ajuste (GOF)

El indicador global de bondad de ajuste GOF, es el más apropiado al realizar el método PLS. Esposito et al. (2008) proponen que el índice de bondad de ajuste global se dé por medio de la raíz cuadrada de la multiplicación de la media aritmética del análisis de la varianza extraída (AVE) y la media aritmética de la varianza explicada (R2) de las variables endógenas o dependientes donde la medida de bondad de ajuste debe variar entre 0 y 1 , a mayor valor, mejor será el índice. Como se puede apreciar en la Tabla 8, el modelo posee un buen ajuste.

Tabla 8: Índice de bondad de ajuste

\begin{tabular}{|l|c|c|c|}
\hline \multicolumn{1}{|c|}{ Constructo } & AVE & $R^{2}$ & GoF \\
\hline Educación Continua & 0,777 & & \\
\hline Calidad de Enseñanza & 0,619 & & \\
\hline Satisfacción del Egresado & 0,641 & 0,587 & \\
\hline Confianza de la Red de Apoyo & 0,836 & & \\
\hline Identificación del Egresado & 0,657 & & \\
\hline Fidelidad del Egresado & 0,508 & 0,405 & \\
\hline Vínculo Relacional del Egresado & 0,654 & 0,531 & \\
\hline Media Aritmética & 0,756 & 0,508 & 0,619 \\
\hline
\end{tabular}

\section{Contraste de hipótesis}

Para analizar el cumplimiento de las hipótesis planteadas es necesario calcular los coeficientes path estandarizados $(\beta)$, en donde sus valores deseables deberían estar por encima de 0,3 , siendo 0,2 un valor mínimo aceptable (Chin, 1998). Por otro lado, el error estándar de los parámetros debe verificar la condición del estadístico $T_{>=}$1,96 (Kwong, 2013). En la Tabla 9, se cumple ambas reglas para todas las relaciones. Dado los resultados anteriores, el modelo queda expresado en la figura 2.

Tabla 9: Contraste de Hipótesis

\begin{tabular}{|c|l|c|c|c|}
\hline Hipótesis & \multicolumn{1}{|c|}{ Relaciones } & Paths $(\beta)$ & Valor T (Bootstrap) & Contraste \\
\hline $\mathrm{H} 1^{+}$ & Educación continua $\rightarrow$ Satisfacción del Egresado & 0,349 & $5,439^{*}$ & Acepta \\
\hline $\mathrm{H} 2^{+}$ & Calidad de enseñanza $\rightarrow$ Satisfacción del E. & 0,592 & $15,178^{*}$ & Acepta \\
\hline $\mathrm{H}^{+}$ & Satisfacción del E. $\rightarrow$ Fidelidad del Egresado & 0,347 & $4,039^{*}$ & Acepta \\
\hline $\mathrm{H} 4^{+}$ & Confianza en la red de apoyo $\rightarrow$ Fidelidad del E. & 0,259 & $2,913^{*}$ & Acepta \\
\hline $\mathrm{H} 5^{+}$ & Identificación del E. $\rightarrow$ Fidelidad del Egresado & 0,322 & $5,128^{*}$ & Acepta \\
\hline $\mathrm{H} 6^{+}$ & Fidelidad del E. $\rightarrow$ Vínculo relacional del Egresado & 0,728 & $28,696^{*}$ & Acepta \\
\hline$* *: t$-value $>1,96$ & & \\
\end{tabular}




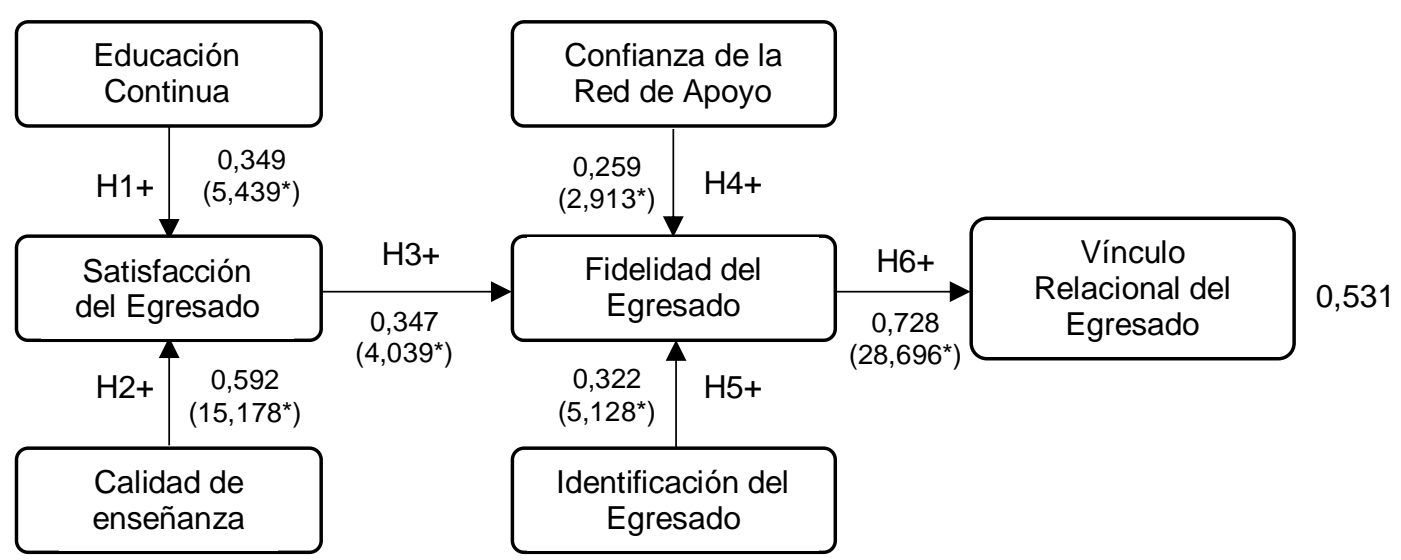

Fig. 2: Modelo de ecuaciones estructurales

\section{DISCUSIÓN}

El trabajo desarrollado verifica y/o evidencia, con confiabilidad estadística, que el vínculo relacional de los egresados de la Universidad de La Serena posee predictores que actúan simultáneamente y que influyen directa e indirectamente en él; educación continua, satisfacción del egresado, calidad de enseñanza, fidelidad del egresado e identificación, que explican en un $53,1 \%$ la varianza de vínculo relacional de los egresados, que es un nivel significativo según Esposito et al. (2008).

En cuanto al análisis bivariante, es posible apreciar que no existe ninguna diferencia significativa según el género de los egresados, pero dada la baja identificación de los egresados con la universidad $(58,50 \%)$ y su nivel medio bajo en satisfacción $(69,21 \%)$, fidelidad $(67,75 \%)$ y vínculo relacional $(63,17 \%)$, se hace evidente la falta de trabajo y compromiso, por parte de la Universidad de La Serena, de mejorar la relación con sus egresados, alimentándola desde que son alumnos, donde la Oficina de Seguimiento de Egresados debe ser líder y gestionar una retroalimentación constante que permita la actualización de las mallas curriculares según los requerimientos del mercado permitiendo a los alumnos aumentar el grado de satisfacción con su alma máter al momento de entrar al campo laboral, motivando a los individuos a promocionar, recomendar, reclutar y mantener una relación a largo plazo (Bhattacharya y Sen, 2003).

En relación a la influencia individual de los constructos sobre el vínculo relacional del egresado, el constructo fidelidad del egresado es el que tiene mayor impacto positivo, seguido por satisfacción, pues presentan un coeficiente estandarizado de 0,728 y 0,592 respectivamente. Esto evidencia con confiabilidad estadística que la fidelidad de los egresados respecto a su alma mater, se va cultivando a medida que transcurre el tiempo transformándose en una relación a largo plazo que incentiva al egresado a seguir vinculado con su institución de educación superior como sugiere Helgesen y Nesset (2007). En este sentido el constructo satisfacción toma un rol fundamental a la hora de fidelizar un estudiante, ya que, un vínculo fuerte entre una universidad y un egresado transforma a este último en un verdadero "embajador" de la institución, recomendándola a su círculo familiar y profesional, permitiendo minimizar el impacto de la fuerte competencia del sector educativo y la frágil situación financiera de las universidades regionales estatales de Chile, transformando este vínculo en una pieza clave de éxito, que posibilita un sistema de mejora continua hacia la excelencia académica, atrayendo nuevos alumnos por medio de las recomendaciones realizadas por egresados, la reelección de la universidad para cursos de postgrado e incluso incentiva la donación hacia la institución (Doña y Luque 2020; Iskhakova et al., 2016). Es por ello que la satisfacción es de vital importancia para la supervivencia de este tipo de instituciones ya que, con una forma fiable de medición, no solo es posible conocer la realidad de la universidad comparándola con la de otros competidores, sino que también, otorga la posibilidad de analizar la institución a lo largo del tiempo, permitiendo mantener siempre los estándares y el objetivo de búsqueda de calidad a todo nivel.

Por último, las demás relaciones a pesar de ser significativas y positivas no poseen valores altos de coeficiente estandarizado, esto indica relaciones más bien débiles, valores entre 0,259 y 0,322 según Chin (1998), esto quiere decir que la universidad debe centrarse en generar procesos de enseñanza y aprendizaje de calidad, impulsar actividades que hagan participes activos a los egresados, ya que de esta forma el ex estudiante se convertirá en un "cliente socio" lo que es consistente con Cabana et al. (2016), que recomienda y vuelve para desarrollarse en postgrados. Estas acciones deben de estar dentro de una metodología cíclica que permita la retroalimentación constante de las diferentes decanaturas, quienes deben de tener como eje central la actualización de mallas curriculares y contenidos según las condiciones del mercado laboral, que dicho sea de paso, se transforma de manera vertiginosa y deja totalmente desactualizadas a las universidades debido 
a sus grandes y pesados procesos burocráticos, dando una sensación negativa no solo a sus alumnos, sino que también a sus egresados, quienes finalmente serán los que mostrarán al mundo la calidad de enseñanza y la imagen de cada institución de educación superior.

\section{CONCLUSIONES}

De los resultados y sus análisis es posible extraer las siguientes conclusiones sobre el vínculo relacional de los egresados con una universidad regional: 1) Es posible confirmar las hipótesis planteadas, es decir, educación continua, satisfacción del egresado, calidad de enseñanza, fidelidad del egresado, confianza en la red de apoyo e identificación del egresado son significativas y están positivamente relacionadas con el vínculo relacional del egresado; 2) La gestión efectiva del modelo propuesto contribuye al posicionamiento estratégico de la ULS y otras Universidades equivalentes, donde sus directivos y la arquitectura organizacional debe cohesionar a toda la comunidad académica para propagar valor en sus estudiantes en pregrado, activo que terminará logrando la satisfacción del egresado. Esto implica asegurarse de cumplir realmente con su perfil de egreso, invirtiendo en tecnologías y procesos que garanticen pasar desde el conocimiento al desarrollo de capacidades demostrables, cualitativa y cuantitativamente; y 3) En una próxima investigación, se debería evaluar la influencia de los desafíos del mercado laboral en la satisfacción del egresado y del estudiante de pregrado, cuestión clave para generar conocimientos que permitan gestionar estratégicamente los procesos de pregrado desde las percepciones balanceadas de un egresado con la "madurez" ganada por su desempeño laboral.

\section{AGRADECIMIENTOS}

Los autores agradecen el apoyo entregado por la Universidad de La Serena para terminar exitosamente esta investigación.

\section{REFERENCIAS}

Ali, F., Zhou, Y., y otros tres autores, Does higher education service quality effect student satisfaction, image and loyalty? A study of International students in Malaysian public universities, https://doi.org/10.1108/QAE-02-2014-0008, Quality Assurance in Education 24(1), 70-94 (2016)

Alvez, H., The measurement of perceived value in higher education: a unidimensional approach, https://doi.org/10.1080/02642069.2011.550042, Service Industries Journal, 31(12), 1943-1960 (2011)

Bhattacharya, C., y Sankar, S., Consumer-company identification: a framework for understaning consumers' relationships with companies, https://doi.org/10.1509/jmkg.67.2.76.18609, Journal of Marketing, 67(2), 76-88 (2003)

Cabana, S., Cortés, F., Vega, D., y Cortés R., Análisis de la fidelización del estudiante de ingeniería con su centro de educación superior: desafíos de gestión educacional, http://dx.doi.org/10.4067/S0718-50062016000600009, Formación Universitaria, 9(6), 93-104 (2016)

Cabana, S., Cortés, F., Aguilera, M., y Vargas, F., Factores determinantes para el intraemprendimiento social: el caso de los estudiantes de ingeniería de la Universidad de La Serena, Chile, http://dx.doi.org/10.4067/S071850062018000200087, Formación Universitaria, 11(2), 87-98 (2018)

Casanoves, J., Küster, I., y Vila, N., ¿Por qué las instituciones de educación superior deben apostar por la marca?, https://doi.org/10.6018/rie.37.1.291191, Revista de Investigación Educativa, 37(1), 111-127 (2019)

Chin, W., The partial least squares approach to structural equation modelling, Lawrence Erlbaum Associates, Estados Unidos (1998)

Doña L., y Luque, T., How loyal can a graduate ever be? the influence of motivation and employment on student loyalty, https://doi.org/10.1080/03075079.2018.1532987, Studies in Higher Education, 45(2), 353-374 (2020)

Douglas, J., McClelland, R., y Davies, J., The development of a conceptual model of student satisfaction with their experience in higher education, doi:10.1108 / 09684880810848396, Quality Assurance in Education, 16(1), 19-35 (2008)

Dutton, J., Dukerich J., y Harquail, C., Organizational images and member identification, https://doi.org/10.2307/2393235, Administrative Science Quarterl, 39(2), 239-263 (1994)

Espinoza, M., Sanhueza, O., Ramírez, N., y Sáez, K., Validación de constructo y confiabilidad de la escala de inteligencia emocional en estudiantes de enfermería, http://dx.doi.org/10.1590/0104-1169.3498.2535, Revista LatinoAmericana de Enfermagem, 23(1), 139-147 (2015)

Esposito, V., Trinchera, L., Squillacciotti, S., y Tenenhaus, M., REBUS-PLS: A response-based procedure for detecting unit segments in PLS path modelling, https://doi.org/10.1002/asmb.728, Applied Stochastic Models in Business and Industry, 34(5), 439-458 (2008)

Falk, R., y Miller, N., A primer for soft modeling, University of Akron Press, Estados Unidos (1992) 
Forcael, E., Vargas, S., Opazo, A., y Medina, L., Rol del ingeniero civil en la sociedad chilena contemporánea, http://dx.doi.org/10.4067/S0718-915X2013000200006, Revista de la Construcción, 12(2), $72-87$ (2013)

Frasquet, M., Calderon, H., y Cervera, A., University-industry collaboration from a relationship marketing perspective: an empirical analysis in a spanish university, https://doi.org/10.1007/s10734-011-9482-3, Higher Education, 64(1), 85-98 (2012)

Gibson, A., Measuring business student satisfaction: a review and summary of the major predictors, https://doi.org/10.1080/13600801003743349, Journal of Higher Education Policy and Management, 32(3), 251-59 (2010)

Gutiérrez, C., Pérez, A., Pérez, M., y Palacios, A., Percepciones de profesores y alumnos sobre la enseñanza, evaluación y desarrollo de competencias en estudios universitarios de formación de profesorado, https://doi.org/10.1174/113564011798392451, Culture and Education, 23(4), 499-514 (2011)

Helgesen, $\varnothing$. y Nesset, E., What accounts for students' loyalty? some field study evidence, https://doi.org/10.1108/09513540710729926, International Journal of Educational Management, 21(2), 126-143 (2007)

Iskhakova, L., Hilbert, A., y Hoffman, S., An Integrative model of alumni loyalty-an empirical validation among graduates from German and Russian universities, https://doi.org/10.1080/10495142.2015.1006490, Journal of Nonprofit y Public Sector Marketing, 28(2), 129-163 (2016)

Johnson, M., Herrmann, A., y Huber, F., The evolution of loyalty intentions, https://doi.org/10.1509/jmkg.70.2.122, Journal of Marketing, 70(2), 122-132 (2006)

Kwong, K., Partial Least squares structural equation modeling (PLS-SEM) techniques using SmartPLS, Marketing Bulletin, 24, 1-32 (2013)

Moriano, J., Topa, G., Valero, E., y Lévy, J., Identificación organizacional y conducta "intraemprendedora", Anales de Psicología, 25(2), 277-287 (2009)

Ruiz, A., y Cruz, F., Las hipótesis de fisher en latinoámerica: un análisis de cointegración, 10.14718/revfinanzpolitecon.2016.8.2.5, Revista de finanzas y política económica, 8(2), 301-326 (2016)

Schlesinger, W., Cervera, A., y Pérez, C., Sticking with your university: the importance of satisfaction, trust, image, and shared values, https://doi.org/10.1080/03075079.2015.1136613, Studies in Higher Education, 42(12), 2178-2194 (2016)

Schlesinger, W., Cervera, A., y Iniesta, M., La identificación egresado-universidad: un modelo desde un enfoque de marketing relacional, Revista de Ciencias Sociales, 18(1), 118-135 (2012)

Schlesinger, W., Cervera, A., y Iniesta, M., Key elements in building relationships in the higher education services context, https://doi.org/10.1080/10496491.2015.1051403, Journal of Promotion Management, 21(4), 475-491 (2015)

Schlesinger, W., Cervera, A., Iniesta, M., y Sánchez, R., Un enfoque de marketing de relaciones a la educación como un servicio: aplicación a la universidad de valencia, http://dx.doi.org/10.15446/innovar.v24n53.43919, Revista Innovar, 24(53), 113-125 (2014)

Scott, S., y Lane, V., A stakeholder approach to organizational identity, https://doi.org/10.2307/259262, The Academy of Management Rewiew, 25(1), 43-62 (2000)

Shams, R., y Belyaeva, Z., Quality assurance driving factors as antecedents of knowledge management: a stakeholderfocussed perspective in higher education, https://doi.org/10.1007/s13132-017-0472-2, Journal of the Knowledge Economy, 10, 423-436 (2019)

Zaheer B., y Rehman, K., A study examining the students satisfaction in higher education, https://doi.org/10.1016/j.sbspro.2010.03.888, Social and Behavioral Sciences, 2(2), 5446-5450 (2010) 
